\title{
MODELING A GEO-SPATIAL DATABASE FOR MANAGING TRAVELERS' DEMAND
}

\author{
Sunil Pratap Singh and Preetvanti Singh \\ Department of Physics and Computer Science, Faculty of Science \\ Dayalbagh Educational Institute (Deemed University) Agra, India
}

\begin{abstract}
The geo-spatial database is a new technology in database systems which allow storing, retrieving and maintaining the spatial data. In this paper, we seek to design and implement a geo-spatial database for managing the traveler's demand with the aid of open-source tools and object-relational database package. The building of geo-spatial database starts with the design of data model in terms of conceptual, logical and physical data model and then the design has been implemented into an object-relational database. The geo-spatial database is developed to facilitate the storage of geographic information (where things are) with descriptive information (what things are like) into the vector model. The developed vector geo-spatial data can be accessed and rendered in the form of map to create the awareness of existence of various services and facilities for prospective travelers and visitors.
\end{abstract}

\section{KEYWORDS}

Geo-spatial Database, Database Design, Conceptual Data Modeling, Object-Relational Database, PostGIS, Travelers' Demand.

\section{INTRODUCTION}

In this digital age, travelers (less dependent on traditional intermediaries) have become sophisticated and more demanding, requesting new sources of information to facilitate the decision making process before accessing services. The information is life-blood of the travel industry [1]. It is necessary, from either internal or external sources, for visiting a destination and for making onsite decisions such as attractions, amenities, transportation, lodging and other facilities nearby. The assessing efficiency of basic services and facilities is multidimensional and includes several factors such as geography, finance, availability and quality. However, the geographic dimension is of great importance which increases the need of managing spatial information. The applications of spatial information system have great social and national relevance and can support governance activities, help in preparing sustainable development strategies, enabling enterprises to better manage business processes and bring geographical knowledge to citizens.

In this paper, we have developed a geo-spatial database for managing the traveler's demand with the aid of open-source tools and object-relational database package. The geo-spatial database is designed to facilitate the storage of geographic information (where things are) with descriptive information (what things are like) into the vector model. The spatial data is derived from existing paper-based map co-ordinates stored in computer file (shapefiles created from color raster base map), whilst the attribute data files are made up of detailed records of any feature or item found on these maps with the items being geo-referenced at their coordinates. The geo-spatial database, 
International Journal of Database Management Systems ( IJDMS ) Vol.6, No.2, April 2014

developed in this study constitutes a great resource to describe and identify the infrastructure elements related to travelers' demand geometrically, thematically and topologically.

\section{STUDY AREA}

Agra, the study area, is a historic city in northern region of India. It is situated between 27.11' degree latitude north and 78.00' degree to 78.20' degree longitude east. The city's rich history, cultural heritage, diversity of religion and healthcare services fascinate a large number of naïve and experienced travelers who visit various places within the city and need spatial information related to destinations, accommodations and other places of interest to improve the convenience and efficiency of travel. The geo-spatial database of city infrastructure, various attractions, public conveniences, transport and other points of interests together with their attribute data will help the travelling community to access, disseminate and visualize the required data in order to better manage their trip.

\section{MATERIALS AND METHODS}

\subsection{Data Modeling}

The development of geo-spatial database starts with the design of data model which shows what data are to be contained in the database and how the items in the database will be related to each other. The database design process is usually divided into three main activities, namely: conceptual, logical and physical data modeling [2].

The data modeling is an abstraction process which focuses on essential elements and ignores nonessential ones with regard to a specific goal [3]. In Figure 1, the different geographic data design models and implementation are shown as the two basic phases involved in the development of a geo-spatial database. There are two common data models for modeling spatial information: fieldbased models and object-based models [4]. The field-based models treat the spatial information as a continuous domain such as altitude, rainfall and temperature. The object-based models treat the information space as if it is populated with recognizable objects that are discrete and spatially referenced. The modeling approach that we take in this study is object-based. It means the database will store a collection of geographical objects that can be modeled by the measurement, properties, and relationships of points, lines, and polygons. Some geographic objects may also have topological properties. 
International Journal of Database Management Systems ( IJDMS ) Vol.6, No.2, April 2014

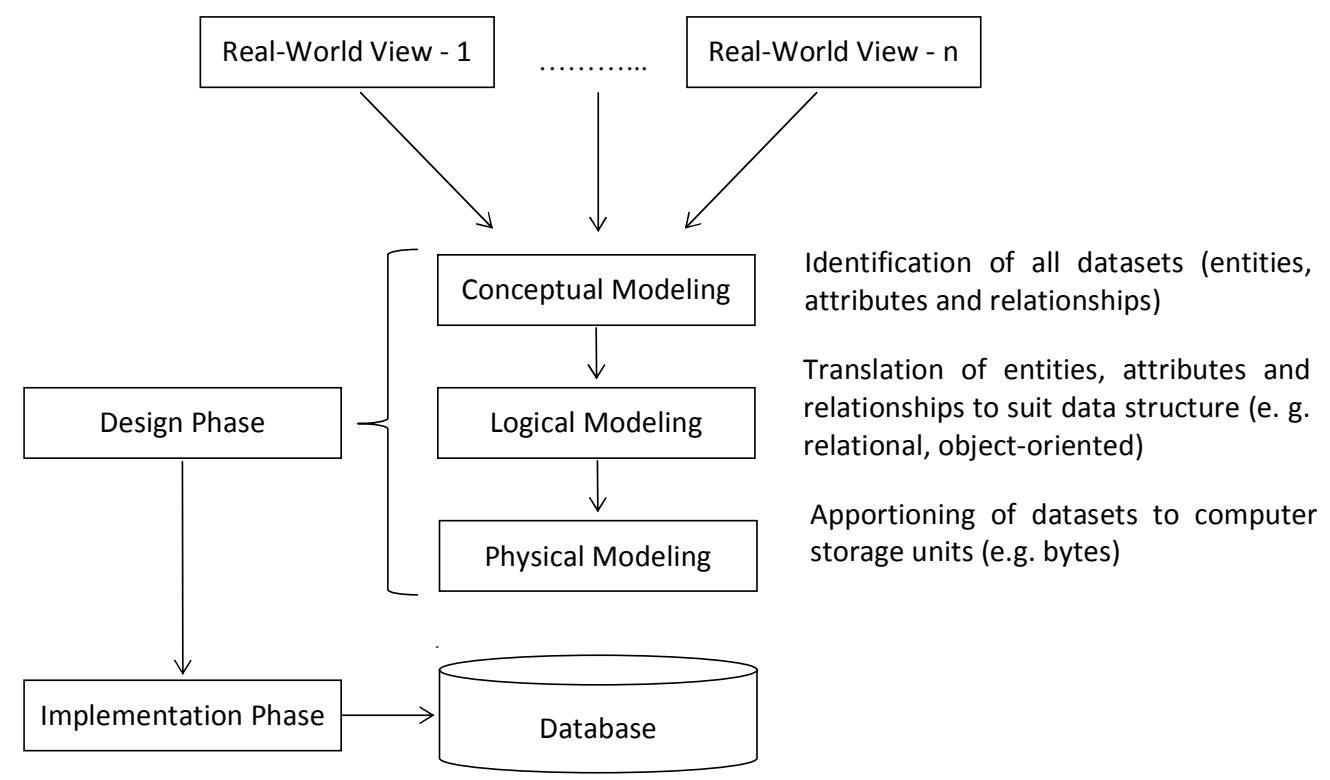

Figure 1. Levels of data view in database development [5].

\subsubsection{Conceptual Modeling}

The conceptual model is a high-level description of how a system is organized and how it operates. In this modeling process, all entities represented as spatial and non-spatial datasets, their attributes and the relationships between them are identified [6].

In order to identify relevant datasets to cater travelers need, the studies [7-11] reveal the importance of spatial and non-spatial information related to transportation, hospitality, major attractions, public conveniences and other places of interest. The key thematic layers to be represented in the database are identified based on these spatial datasets. These layers include green patches, wetlands, attractions, amenities and transportation infrastructures which are further categorized into basic entities; for example, the green patch layer is decomposed into parks, gardens and forests. 
International Journal of Database Management Systems ( IJDMS ) Vol.6, No.2, April 2014

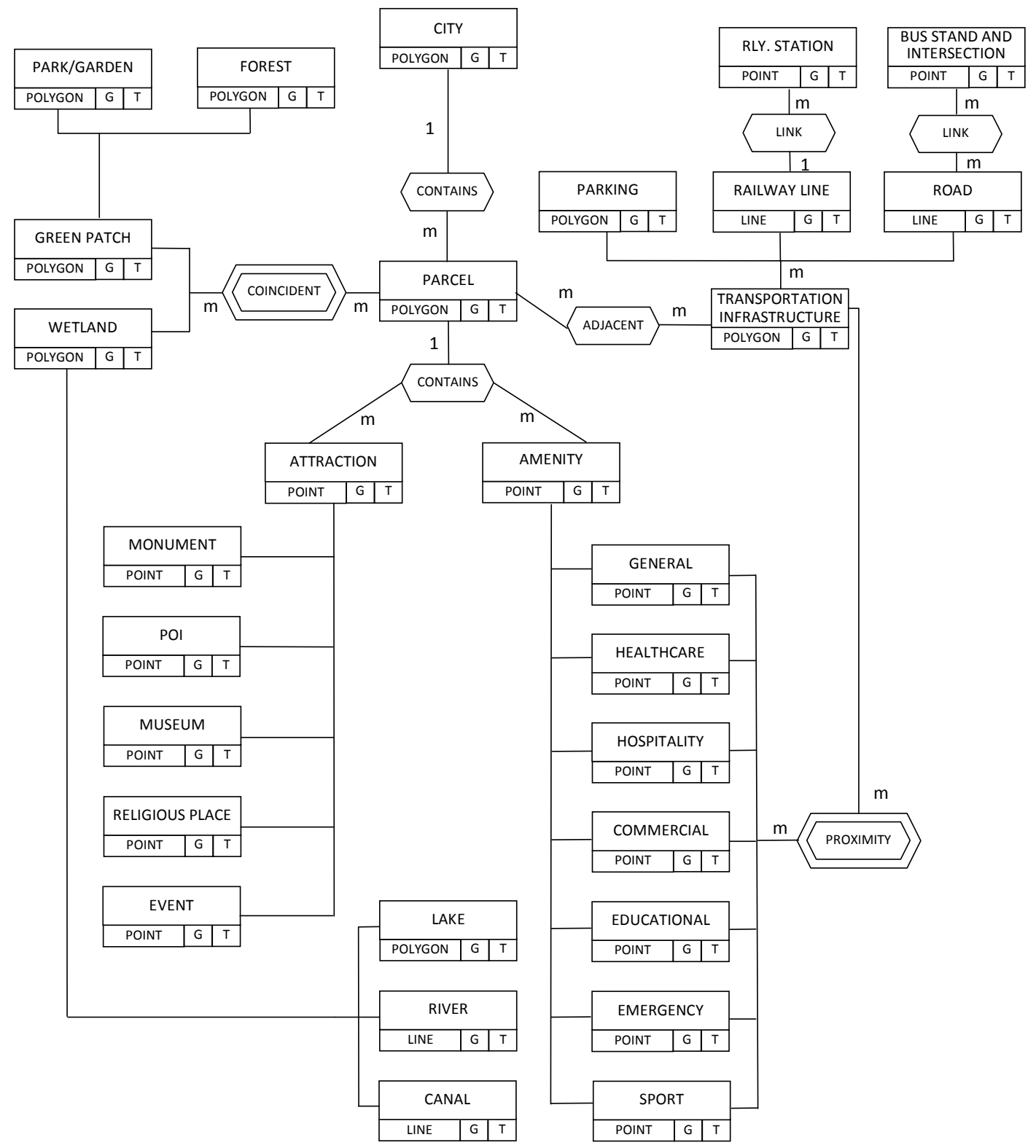

Figure 2. Conceptual data model for managing travellers demand.

The conceptual modeling in database design often makes use of a formal approach known as entity-relationship (E-R) modeling [12]. It is a graphical method of representing entities, all important relationships between the entities, and all attributes of either entities or relationships which must be captured in the database. There are several dialects of E-R formalism [12-15] and also many adaptations have been made for specific needs like modeling of geographic phenomenon. In this study, we have used Calkins' [15] extension to basic entity-relationship data models for modeling our geo-spatial database. In its extended symbology, entity symbol contains the name of the entity and additional information indicating the corresponding spatial entity (point, line or polygon), a code indicating topology, and a code indicating encoding of the spatial entity by coordinates. The spatial relationships are handled by three relationship symbols: the traditional diamond symbol for normal database relationship; an elongated hexagon and a double elongated hexagon to represent spatial relationship. The elongated hexagon represents spatial 
relationships through topology (connectivity and contiguity) and the double elongated hexagon uses $\mathrm{x}$, y coordinates as well as related spatial operations (coincidence, containment and proximity) for defining spatial relationships.

The entity-relationship diagram in Figure 2 represents the proposed conceptual model of geospatial database. The attributes are not shown in the diagram for readability purpose; however they have been identified and included in its corresponding logical model.

\subsubsection{Logical Modeling}

The logical data model expresses a conceptual data model in terms of (software) computable data constructs (entity classes or object classes), operations (create relationships) and validity constraints (rules) [16]. In this section, the description of feature classes and attributes is translated into an object-relational database model (an implementation data model), thus mapping the high-level data model into the implementation model of the database management system (DBMS). Figure 3 illustrates the fully attributed Road-Junction logical model, a part of Figure 2.

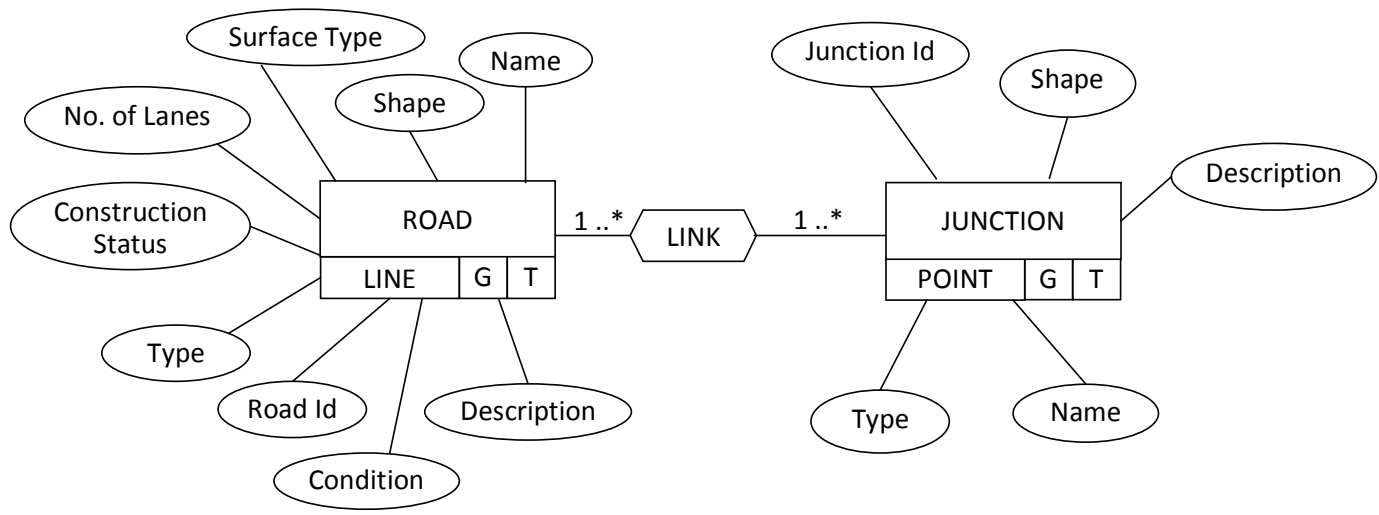

Figure 3. Logical data model for Road-Junction relationship.

\subsubsection{Physical Modeling}

Physical modeling involves the actual design of a database according to the requirements that were established during logical modeling. The data type implementation and the indexing of data type fields are specified at this level of modeling. Each entity is transformed into a database table, and for each relationship foreign keys are defined. This model is a fully-attributed data model that is dependent upon a specific version of a data persistence technology.

In this phase, the logical data model developed in Figure 3 has been transformed to physical data model (Figure 4) using the syntax of PostgreSQL/PostGIS, which follows the object-relational database design. 
International Journal of Database Management Systems ( IJDMS ) Vol.6, No.2, April 2014

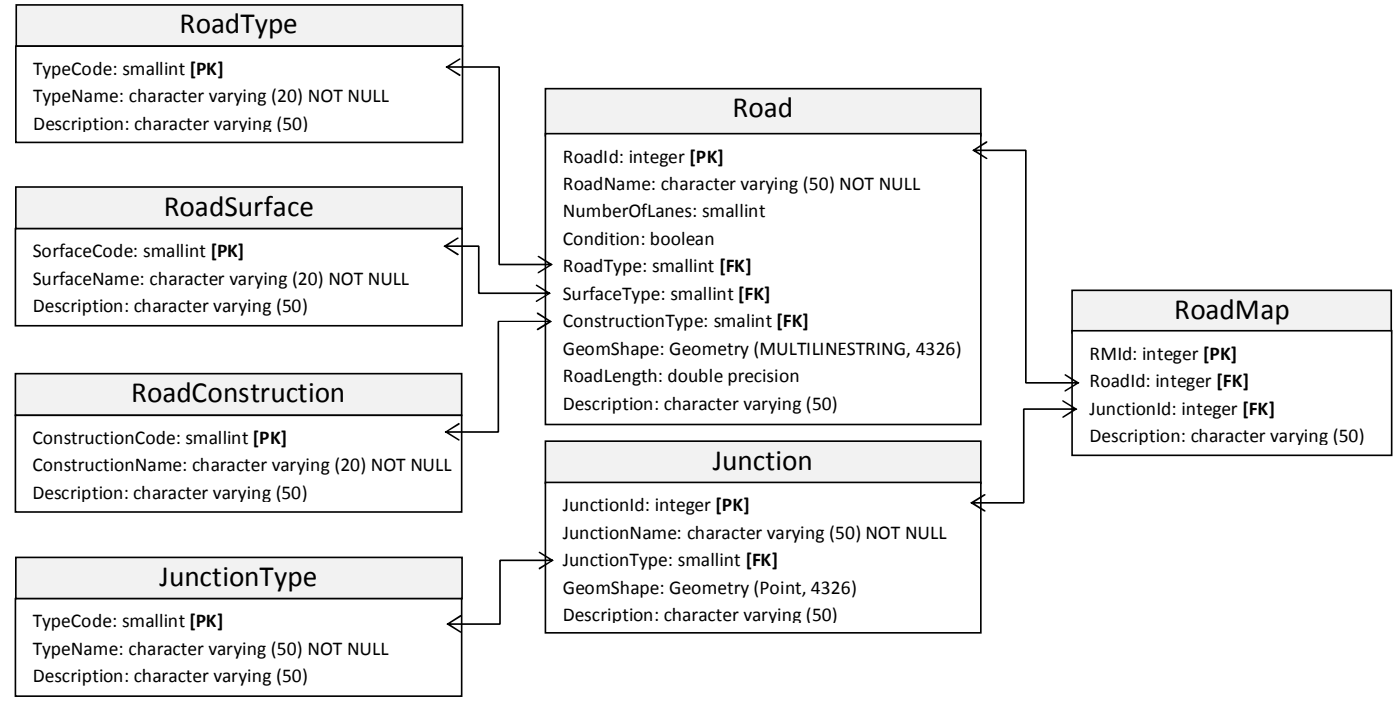

Figure 4. Physical data model (transformed from Figure 3).

\subsection{Implementation}

The analysis on spatial data can be performed well if the data of geographic objects is stored in relational database. In this study, PostgreSQL is chosen as the spatially-enabled object-relational database management system (ORDBMS), which presents object-oriented features as extension of the relational model [17] and provides scalability and support for complex data types (large objects, multimedia data, spatial data, etc.) by combining both the relational and object-oriented models.

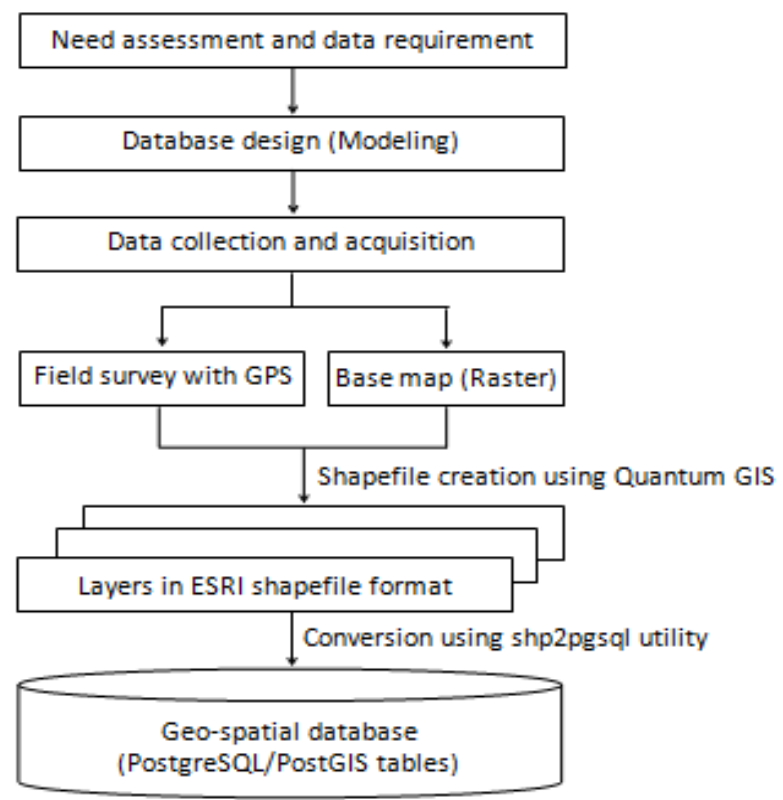

Figure 5. Flow diagram of spatial database development.

It is not easy to store the spatial data in a standard RDBMS, thus spatial extensions have been developed and standardized by the OGC (Open Geo Consortium). PostGIS is an open-source and OGC compliant spatial database extender for PostgreSQL [18]. It adds spatial functions and 
International Journal of Database Management Systems ( IJDMS ) Vol.6, No.2, April 2014

specialty geometry data types to the database. It is an excellent way to bring tabular and spatial data together into a common management environment. The security of this data storage can be ensured by following the approach discussed in [19].

The geospatial data describing the study area is loaded from shapefiles (ESRI's proprietary storage format for geospatial data) which were created from city's base map (color raster map obtained from Survey of India). Quantum GIS (an open-source desktop mapping engine) is used for geo-referencing the base map and creating separate vector layers (shapefiles) for different geometry types. The use of free and open-source geospatial tools results in a rapid implementation with minimal or no software input cost [20]. The conversion of shapefiles to PostgreSQL database tables is achieved using the shp2pgsql utility included as part of the PostGIS extension (as shown in Figure 5). In each table, the "GeomShape" column (used to store vector geospatial data) has been included in a special PostGIS table "geometry_columns". This table defines: all other tables containing geometry columns, spatial dimension of geometry columns, SRID of the spatial reference system used for coordinate geometry, and the type of spatial object (POINT, LINESTRING, POLYGON, etc.).

The spatial reference systems which could be assigned to the geospatial data are defined in another special PostGIS table named "spatial_ref_sys". This table lists a large number of spatial reference systems and details needed to transform between them. For all database tables (storing geographic objects), we have filled PostGIS SRID 4326 which corresponds to WGS84 spatial reference system.

\section{RESULTS AND DISCUSSION}

The geo-spatial database, developed in this study constitutes a great resource to describe and identify the infrastructure elements related to travelers' demand geometrically, thematically and topologically. The developed database constitutes an adequate support platform to implement an integrated spatial information system by:

- storing and manipulating simultaneously the geometry and attribute information

- providing an array of analytic methods to quickly process spatial and statistical information.

- managing different user roles giving different access to and production of information by various stakeholders involved in travel and tourism planning process.

- accessing information locally or remotely over a network for manipulation and visualization.

- storing large quantities of information and providing access to selective data through queries in an efficient way.

- managing information in a single central repository.

The establishment of geo-spatial database will serve as a valuable tool for effective decision making in spatial analysis (by means of spatial measurement and spatial predicate), spatial modeling, queries and network analysis. Moreover, the availability of comprehensive spatial information can provide easier access to distance information, neighboring locations and other information of travelers' interest.

\section{CONCLUSION}

This paper describes the development of a geo-spatial database using open-source tools and object-relational database package. The geo-spatial database facilitates the storage of geographic information (where things are) with descriptive information (what things are like) into the vector 
International Journal of Database Management Systems ( IJDMS ) Vol.6, No.2, April 2014

model. The development of geo-spatial database consists of two phases: design (data modeling) and implementation. The modeling phase is concerned with what data are to be contained in the database and how the items in the database will be related to each other. In this phase, the geographic information relevant to travelers' demand is identified in the form of spatial and nonspatial datasets. These datasets are then modeled into conceptual, logical and physical data models successively. In implementation phase, the geo-spatial data describing the study area is loaded into PostgreSQL/PostGIS tables. These database tables are populated from shapefiles (created from city's base map), GPS field survey and other published/unpublished data sources available on Internet. It is envisaged that the geo-spatial database for the city would greatly benefit the travelling community and citizens to serve the basic need by providing access to geospatial information and geo-enabling decision making process.

\section{ACKNOWLEDGEMENT}

The corresponding author is thankful to University Grants Commission, India for providing financial support in the form of research fellowship under RFSMS scheme.

\section{REFERENCES}

[1] Sheldon, P. J. (1993) "Destination Information Systems", Annals of Tourism Research, Vol. 20, pp. 633-649.

[2] Elmasri, R. \& S. B. Navathe (1989) Fundamentals of Database Systems, CA, USA: Benjamin/Cummings Publishing.

[3] Bedard, Y. \& F. Paquette (1989) "Extending entity/relationship formalism for spatial information systems", in Proc. of the $9^{\text {th }}$ International Symposium on Computer-Assisted Cartography (AutoCarto 89), Maryland, USA, pp. 818-827.

[4] Shekhar, S., Chawla, S., Ravada, S, Fetterer, A., Liu, X. \& C. Lu (1999) "Spatial Databases Accomplishments and Research Needs", IEEE Trans. On Knowledge and Data Engineering, Vol. 11, pp. 45-55.

[5] Akinyemi, F. O. (1999) "Database design and development for census mapping in Nigeria", in Balogun, O. Y. \& S. S. O. Soneye, (Eds.): Cartography in the Service of Government, Nigeria, Lagos: Nigerian Cartographic Association, pp. 191-202.

[6] Akinyemi, F. A. (2010) “Conceptual Poverty Mapping Data Model”, Transactions in GIS, Vol. 14, pp. 85-100.

[7] Duran, E.; Seker, D. Z. \& M. Shrestha (2004) "Web based information system for tourism resort: A case study for side/Manavgat", in Altan, O. (Eds.): XX ${ }^{\text {th }}$ ISPRS Congress Youth Forum, Istanbul, Turkey, pp. 90-93.

[8] Kumar, P., Singh, V. and D. Reddy (2005) "Advanced Traveler Information System for Hyderabad City”, IEEE Transactions on Intelligent Transportation Systems, Vol. 6, pp. 26-37.

[9] Fajuyigbe, O. Balogun, V. F. \& O. M. Obembe (2007) "Web-Based Geographical Information System (GIS) for Tourism in Oyo State, Nigeria”, Information Technology Journal, Vol. 6, pp. 613622.

[10] Jovanovic, V. \& A. Njegus (2008) "The Application of GIS and ITS Components in Tourism", Yugoslav Journal of Operations Research, Vol. 18, pp. 261-272.

[11] Alqeed, M. A., Bazazo, I. K., Hasoneh, A. I. \& B. A. Alqaid (2010) "Using Geographic Information System to Visualize Travel Patterns and Market Potentials of Petra City in Jordan" International Journal of Marketing Studies, Vol. 2, pp. 144-159.

[12] Ullman, J. D. (1988) Principles of Database and Knowledge-Base Systems Vol. 1: Classical Database Systems, NY, USA: Computer Science Press. 
International Journal of Database Management Systems ( IJDMS ) Vol.6, No.2, April 2014

[13] Martin, J. \& C. McClure (1988) Structured Techniques: The Basis for CASE, Revised Edition, NJ, USA: Prentice-Hall.

[14] Spencer, R., Teorey, T. \& E. Hevia (1990) "ER Standards Proposal", in Proc. of the $9^{\text {th }}$ International Conference on Entity-Relationship Approach, Lausanne, Switzerland, pp. 405-412.

[15] Calkins, H. W. (1996) "Entity Relationship Modeling of Spatial Data for geographic Information Systems”, International Journal of Geographic Information Systems, Vol. 10, pp. 1-19.

[16] University of Washington. http://faculty.washington.edu/nyerges/gisdbp/GISDBP_chapter_1_v18.pdf (accessed on 14.10.13).

[17] Sabău, G. (2007) "Comparison of RDBMS, OODBMS and ORDBMS", Informatica Economica, Vol. XI, pp. 83-85.

[18] PostGIS. http://postgis.net/ (accessed on Nov. 8, 2013).

[19] Chaudhary, S. Z. \& P. Venkatachalam (2013) "Conceptual Framework for Geospatial Data Security”, International Journal of Database Management Systems, Vol. 5, pp. 29-35.

[20] Singh, S. P. \& P. Singh (2014) "Mapping Spatial Data on the Web using Free and Open-Source Tools: A Prototype Implementation”, Journal of Geographic Information System, Vol. 6, pp. 30-39.

\section{Author's Biography}

Sunil Pratap Singh is a Research Scholar in the Department of Physics and Computer Science, Dayalbagh Educational Institute (Deemed University), Agra, India. He received his Master degree in Computer Applications from Uttar Pradesh Technical University, Lucknow (India) in 2008. His research interests include decision support system (DSS), geographical information system (GIS), web and mobile based application development.

Preetvanti Singh is an Associate Professor in the Department of Physics and Computer Science, Dayalbagh Educational Institute (Deemed University), Agra, India. She received her Ph. D. degree in Operation Research from Dayalbagh Educational Institute, Agra, India. Her current research interests include decision support system (DSS), geographical information system (GIS) and optimization techniques. She also co-authors computer science textbooks. 